Internist 2013 $\cdot$ 54:1041-1042

DOI 10.1007/s00108-012-3226-z

Online publiziert: 30. August 2013

(c) Springer-Verlag Berlin Heidelberg 2013

M. Hallek · M. von Bergwelt-Baildon

Klinik I für Innere Medizin, Universitätsklinikum Köln

\title{
Onkologische und hämatologische Notfälle
}

\section{Intensivmedizinisches Management}

Hinsichtlich der intensivmedizinischen Betreuung von Patienten mit malignen Grunderkrankungen bestehen erhebliche konzeptionelle Unterschiede zwischen den operativen Fächern und der Inneren Medizin. Aufgrund der häufig notwendigen postoperativen intensivmedizinischen Überwachung werden alle Tumorpatienten, die einer chirurgischen Intervention unterzogen werden können (kurativ oder palliativ), abhängig von der Prognose der Grunderkrankung intensivmedizinisch betreut. Darüber hinaus werden in der Regel alle Patienten, bei denen in der Folge einer solchen Operation ernsthafte Komplikationen auftreten, auch im fortgeschrittenen Tumorstadium intensivmedizinisch versorgt. Demgegenüber ist eine Intensivaufnahme wegen internistischer Krankheitsbilder in vielen Krankenhäusern durch die Nebendiagnose Krebs erheblich erschwert, wenn nicht sogar unmöglich. Woran liegt das?

\section{》) Die Nebendiagnose Krebs erschwert in vielen Kliniken die Intensivaufnahme}

Handelt es sich um primär in der internistischen Onkologie versorgte Patienten, so liegen fortgeschrittenere Erkrankungsstadien und limitiertere Prognosen als bei chirurgischen und damit operablen Patienten vor. Die oben geschilderte Problematik besteht allerdings auch bei internistischen Patienten, die nicht primär wegen Tumorerkrankungen versorgt werden, z. B. im Falle der Aufnahmediagnose Myokardinfarkt. Wenn auch in historischen Kollektiven das Überle- ben onkologischer Patienten auf der Intensivstation (ICU) mit Zwei- oder Multiorganversagen $<20 \%$ lag, so zeigen doch neuere Studien, dass spezialisierte Zentren inzwischen Überlebensraten von $60-70 \%$ erreichen, trotz Beatmung und Mehrorganversagen. In einer Studie von Azoulay et al. [Clin Oncol 2013, 31(22):2810-2818] lebte von Tumorpatienten, denen aufgrund der Einschätzung „zu schlecht für eine Intensivversorgung" die ICU-Aufnahme versagt blieb, nach 180 Tagen noch jeder Fünfte. Nach unserer Einschätzung ist eine dringende Überprüfung und Standardisierung der Indikationsstellung zur intensivmedizinischen Versorgung und der Managementstrategien kritisch kranker Tumorpatienten von Nöten.

Diese Notwendigkeit resultiert insbesondere aus folgenden Entwicklungen:

- Die Prävalenz von Krebserkrankungen und die damit verbundene Lebenserwartung steigen stetig an. Sekundär führt dies auch zu einer $\mathrm{Zu}$ nahme an kritisch kranken Krebspatienten. Inzwischen liegt bei 15$20 \%$ aller ICU-Patienten eine Krebserkrankung vor.

- Historische Altersgrenzen zur Einleitung von Chemotherapien werden vermehrt hinterfragt. So werden in vielen großen Zentren ausgewählte Patienten mit akuten Leukämien (bis in die 8. Lebensdekade) einer Induktionschemotherapie oder allogenen Stammzelltransplantation zugeführt.

- Aufgrund der besseren Supportivtherapien ist auch bei Patienten mit hoher Komorbidität die behandlungsassoziierte Mortalität relativ niedrig.
- Eine Reihe neuer Medikamente in der Hämatologie und Onkologie zeigen teils erhebliche akute Nebenwirkungen wie Zytokinsturm oder ein Tumorlysesyndrom.

Aufgrund dieser Entwicklungen nimmt die Zahl kritisch kranker Tumorpatienten mit prinzipiell notwendiger intensivmedizinischer Versorgung stetig zu. Sie bedürfen in Bereichen der Hochdosischemotherapie, der Behandlung der akuten Leukämien, der allogenen Stammzelltransplantation und des Einsatzes immunmodulatorischer Substanzen dringend intensivmedizinischer Unterstützung.

Nach unserer Einschätzung gibt es mindestens drei klinische Szenarien, für die internistische Intensivmediziner und Onkologen gemeinsam differenzierte intensivmedizinische Konzepte entwickeln müssen:

- Potenziell kurativ behandelbare maligne Systemerkrankungen, u. a. Leukämien, Hodentumoren und Sarkome

- Nicht kurativ behandelbare Tumorerkrankungen mit mehrjähriger Lebenserwartung

- Tumorerkrankungen im streng palliativen Setting, die mit einer mehrwöchigen oder mehrmonatigen Lebenserwartung einhergehen

Am Beispiel dieser drei Szenarien muss geprüft werden, ob es Indikatoren gibt, die bei der Einschätzung helfen, für welche Patienten ein sog. ,full-code management" bzw. ein „ICU trial“ (mehrtägige Behandlung mit Reevalution) sowie eine palliative Aufnahme auf die Intensivsta- 
tion zum Symptommanagement, z. B. bei oberer Einflusstauung oder Atemwegskompression, in Betracht kommt.

Eine wichtige Grundlage ist bei einer solch hohen Frequenz an Patienten mit malignen Grunderkrankungen, dass jeder Intensivmediziner mit dem Management onkologischer Notfälle vertraut ist. Im aktuellen Schwerpunkt konzentrieren wir uns daher auf häufige - und auch über den Rahmen der Inneren Medizin hinaus relevante - Erkrankungen, wie die tumorassoziierte Hyperkalzämie, die Leukostase und die Tumorlyse.

\section{》) Jeder Intensivmediziner sollte mit dem Management onkologischer Notfälle vertraut sein}

Des Weiteren werden die Besonderheiten von Infektionen bei Tumorpatienten und das Management der neutropenen Sepsis diskutiert. Die thrombotischen Mikroangiopathien sind Querschnittserkrankungen der Hämatologie, Transplantationsmedizin und Nephrologie, die fächerübergreifend verstanden und behandelt werden sollten. Abschließend werden generelle Konzepte und Besonderheiten im Umgang mit onkologischen Patienten in der Intensivmedizin erläutert.

Wir hoffen, mit dieser Schwerpunktausgabe einen Beitrag dazu zu leisten, dass kritisch kranke hämatologische und onkologische Patienten, soweit indiziert, eine optimale internistisch-intensivmedizinische Behandlung erfahren.

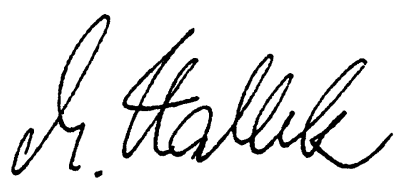

M. Hallek

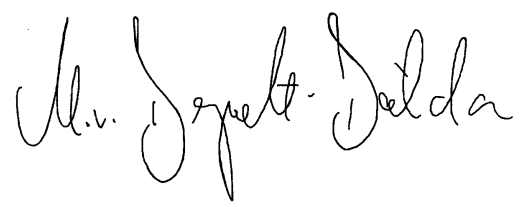

M. von Bergwelt-Baildon
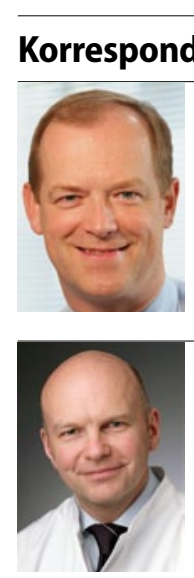

Prof. Dr. Dr.

M. von Bergwelt-Baildon

Klinik I für Innere Medizin,

Universitätsklinikum Köln

Kerpener Str. 62, 50937 Köln

michael.bergwelt@uk-koeln.de

\section{Einhaltung ethischer Richtlinien}

Interessenkonflikt. M. Hallek und M. von BergweltBaildon geben an, dass kein Interessenkonflikt besteht.

\section{Ausschreibung Theodor-Frerichs-Preis 2014 Deutsche Gesellschaft für Innere Me- dizin e.V.}

Die Deutsche Gesellschaft für Innere Medizin e.V. (DGIM) verleiht jährlich anlässlich ihrer Jahrestagung den Theodor-FrerichsPreis für die beste vorgelegte, möglichst klinisch-experimentelle Arbeit auf dem Gebiet der Inneren Medizin. Der Preis ist mit EUR 30.000,- dotiert.

Die Arbeit in deutscher oder englischer Sprache darf in der vorliegenden Form nicht vor dem 15. Oktober 2012 veröffentlicht worden sein. Sie darf nicht zeitgleich an einem ähnlichen Wettbewerb teilnehmen oder bereits mit einem entsprechenden Preis ausgezeichnet worden sein. Das Alter des Verfassers sollte 40 Jahre nicht überschreiten. Bewerber müssen Mitglied der DGIM sein. An der Arbeit können auch mehrere Autoren beteiligt sein; in diesem Falle wird erwartet, dass sich die Arbeitsgruppe auf einen Wissenschaftler einigt, der als persönliche Bewerberin oder persönlicher Bewerber auftritt.

\section{Bewerbung:}

Bewerber werden gebeten, ihre Arbeit in fünffacher Ausfertigung unter Angabe eines Kurztitels, der Anschrift und des Geburtsdatums zusammen mit einem kurzen Curriculum vitae bis zum 15. Oktober 2013 an den Generalsekretär der DGIM Herrn Prof. Dr. med. Dr. h.c. Ulrich R. Fölsch Irenenstraße 1

65189 Wiesbaden unter Beifügung einer einseitigen Zusammenfassung einzureichen.

Die Verleihung des Preises erfolgt anlässlich der festlichen Abendveranstaltung der 120. Tagung der DGIM am 27. April 2014 in Wiesbaden. Die Bewerber werden gebeten, an der festlichen Abendveranstaltung im Kurhaus Wiesbaden teilzunehmen.

\section{Kontakt}

Prof. Dr. med. Dr. h.c. Ulrich R. Fölsch

Generalsekretär

Irenenstraße 1

65189 Wiesbaden

Tel. (0611) 2058 040-0

Fax: (0611) 2058 040-46

info@dgim.de, www.dgim.de

www.dgim2014.de 